Fantasies of medical reality: an observational study of simulation-based medical education

\author{
Author contact details: \\ Dr. Caroline Pelletier \\ Department of Lifelong and Comparative education \\ Institute of Education \\ University of London \\ 20 Bedford Way \\ London WC1H 0AL \\ c.pelletier@,ioe.ac.uk \\ Professor Roger Kneebone \\ Faculty of Medicine, Department of Surgery and Cancer \\ Imperial College \\ Clinical Skills centre \\ Paterson Wing \\ St Mary's Campus \\ W2 1 PG \\ r.kneebone@imperial.ac.uk
}

Word count excluding references: 7731

Word count including references: 8689 


\section{Fantasies of medical reality: an observational study of simulation-based medical education}

Medicine is increasingly taught in immersive simulated environments, to supplement the apprenticeship model of work-based learning. Clinical research on this educational practice focuses on its realism, defined as a property of simulation technology. We treat realism as a function of subjective but collectively organised perception and imbued with fantasy, which we define by drawing on Lacanian studies of virtual reality and workplace organisation. Data from an observational study of four simulation centres in London teaching hospitals is drawn on to present an account of what was taught and learned about medicine, including medical failure, when medical practice was simulated.

Keywords: Simulation, fantasy, technology, virtual reality, work, medical education 
This paper is about a relatively new practice in medical education called 'high-fidelity' or 'immersive' simulation, and how it looks when it is seen through the concept of fantasy. Our interest in seeing it through this lens derives from the term 'fidelity', which describes simulation on the basis of its faithfulness to reality (Issenberg et al, 2005). The term opens a gap between an original and its forgery, which is usually explained technologically (CMO, 2008; Gaba, 2004), and thus as known and measurable, in that it is the difference between two stable entities. The term fidelity thus marks a difference between simulation and reality, foreclosing the possibility of seeing simulation as real: part of reality and also creating it, by signifying clinical events. Yet simulating an object - such as the gastro-intestinal system - or a situation - such as a woman in hospital because her mother is dying - involves making sense of it; understanding, explaining and feeling it, and such that one can intervene on the basis of such sensibilities and narratives (Johnson and Berner, 2012; McNaughton, 2012; Taylor, 2011; Johnson, 2008; Waldby, 1997). A simulation of a medical phenomenon thus stages how it assumes form and meaning, experientially; how it appears realistic. In that the term fantasy accounts for how the world comes to be treated as realistic - meaningful, credible, an experience one can have faith in and be loyal to -, its recruitment to explore simulation-based medical education serves to highlight how this pedagogic practice is implicated in generating attachments to and identifications with ways of seeing, feeling and understanding medical work and phenomena.

Let us first describe what we mean by high-fidelity simulation and why we are concerned with it. Over the last ten years, many teaching hospitals internationally have had 
education facilities built which feature elements constituting operating theatres and hospital wards, such as beds, drug cabinets, and gas outlets. These facilities also include manikins that produce physiological responses such as a palpable pulse rate, breathing sounds, and monitor readouts.
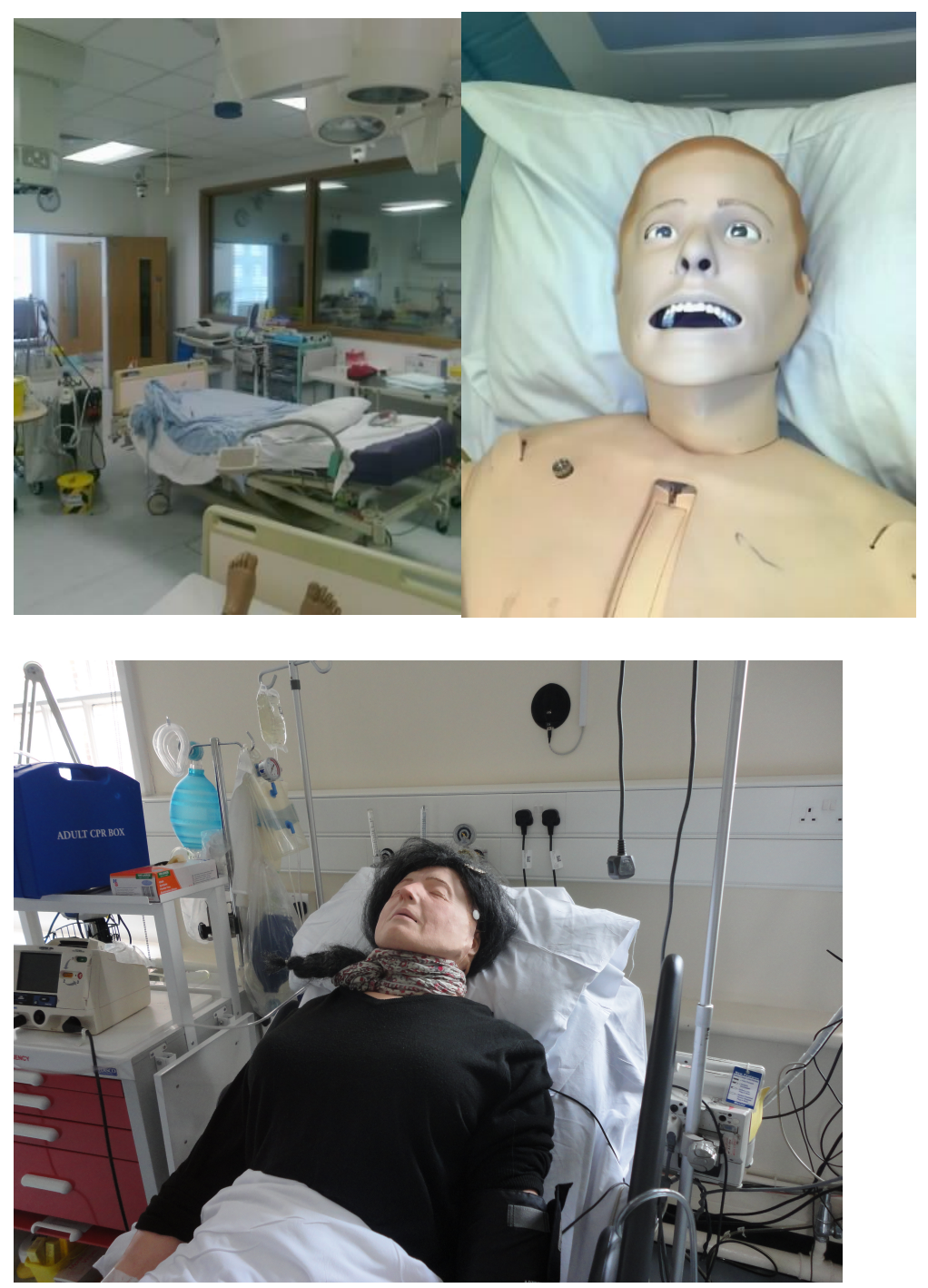

[insert figure 1 - Simulation suite, showing simulated ward and one way mirror] [insert figure 2 - 'High-fidelity' manikin for anesthetic training] [insert figure 3 - 'High-fidelity’ manikin for communication skills training] 
Although simulation has always featured in medical education - for example, chicken meat has long been used to simulate human flesh in teaching suturing, or actors used to simulate distressed patients in teaching how to break bad news - these new 'high-fidelity' facilities respond to more recent concerns about the quality and range of work-based learning (CMO, 2008). They are intended to act a proxy for, or supplement to, the traditional medical apprenticeship, by making it possible to simulate not only isolated tasks but also the complexity of the clinical setting, "including its social and emotional dimensions" (Curran, 2010).

The ethics of 'high-fidelity' simulation are usually articulated in the language of patient safety: "mistakes made during simulated exercises do not cause harm to living patients and can be more easily exposed and discussed. Mishaps in the course of learning can thus be reviewed openly without concern of liability, blame, or guilt—even decisions and actions that result in the death of the simulated patient. SBME [simulation-based medical education] can help break the culture of silence and denial in medicine regarding untoward outcomes and mistakes and their implications about the learner's competence" (Ziv et al, 2003 p.785). The quote illustrates how simulation is treated as ethical insofar as it enables inexperienced doctors to gain experience without harming patients, and that it does this without the negative emotions associated with learning through trial and error.

The term fantasy gives shape to the trouble we have with this argument. It should be emphasized that we are not, consequently, against simulation. Rather, our experience of simulation-based teaching, as well as the observational study which is the main focus in 
this article, lead us to question the ethics of treating patients as in need of protection from incompetent doctors, and doctors from the negative emotions consequent upon learning medicine. Such claims distribute risk, danger and safety to distinct places in a vision of what doing and learning medicine involves, making some aspects invisible, abject, outside the frame of signification (Rancière, 2004). Treating simulation as affecting and affective in this way allows us to see how the practice is itself 'emotional' or invested with attachments and identifications, including the (partial) position from which a medical scene is modeled. Such a perception has political and methodological implications, troubling the treatment of reality as given; indeed, the term fidelity makes reality subject to agreement, with disagreement a consequence either of misunderstanding or incomprehension (Rancière, 2004a). Differences in visions of reality are then cast as temporary delays until consensus is re-established, for instance, by better medical evidence, technology or practice. Pedagogically, simulation is then also treated as an anteroom prior to entry into a consensual community of experts and an innocent order of reality (Pelletier, 2012). Highlighting the fantasmatic aspects of simulation opens up possibilities for making the order of medical reality appear multiple, rather than ranging from inferior to superior, or low to high in fidelity. It also makes medical reality appear alterable, open to re-organization, rather than apparently imposed by the facts of a situation (Pelletier, 2009; Rancière, 1987).

To summarise our aim in this article then: we are looking to close the gap between the authentic original and the inauthentic forgery, and open one instead between the real and its sensibility, such that disagreements about whether a simulation is realistic or not can 
be heard not simply as differences in levels of knowledge but rather as fantasmatic attachments to different versions of medicine. This seems important in exploring how simulation can be implicated in more democratic healthcare, including in sensibilities of what constitutes failure (Paget 2004).

\section{What is fantasy and how do we know?}

Our ideas about fantasy are informed by Lacanian approaches, in which fantasy is constitutive of knowledge, rather than its opposite; and in which it is accounted for by looking at practices of signification, rather than, for instance, biological instinct. This makes fantasy a socio-cultural phenomenon; that which lends the real an imaginary appearance of meaning by signifying it in distinct ways.

In Lacanian accounts, the reason for fantasy is to give desire a goal; to answer the question of what I desire, and what others desire of me (Nusselder, 2009; Zizek, 1989): "[fantasy] provides a schema according to which certain positive objects in reality can function as objects of desire" (Zizek, 1997 p.7). In discussing how reality assumes its appearance, Lacan (2007 p.47) evokes the selectivity of both fantasy and perception, phenomena which are intertwined in his writings: "something sifts, sieves, in such a way that reality is only perceived by man, in his natural, spontaneous state at least, as radically selected. Man deals with selected bits of reality". Selection is realized to make desire sustainable - in the sense of both bearable and renewable - and by implication to make the desiring/desired subject also intelligible, sensible. 
This brief attempt to pin down the notion of fantasy does not do justice to the complex, multiple ways in which the concept is articulated in Lacan's work and Lacanian writing, and gallops roughshod over important distinctions in Lacanian approaches which are undone in selective quotes, such as between the real and reality. This is (partly) intentional, or performative, to bring out the relationship between fantasy, language, identification, desire, and subjectivity, including in the work of distilling a Lacanian formula for fantasy so as to make sense of various experiences, and be made sense of as (likeable, intelligible - by the right people) researchers. Our argument is intended here to make apparent that fantasy is not 'out there' in other people's minds, but intrinsic to collective sense-making, including research, which also gives desire a goal, such as 'more democratic healthcare' (Lapping, 2013).

In exploring how the practice of simulation can be researched in its fantasmatic dimension, we have drawn on two main bodies of research.

\section{Critical business studies}

The first is critical business and organization studies, in which a Lacanian conception of fantasy is deployed to describe how employees assume the desires and agendas articulated by the organization as their own, and in so doing, become recognizable to themselves and to others as (good, useful, high-performing, flexible, etc.) employees (Cederstrom and Hoedemakers, 2010; Glynos, 2011, 2010, 2008; Hoedemakers, 2009; Stavrakakis, 2010, 2008). Fantasy here is aligned with ideology, a phenomenon 
employees invest in to secure a position for themselves within a meaningful scene: "the logic of fantasy, then, can be construed as a narrative affirmed by workers, often unconsciously, preventing the contestation of suspect social norms, and making less visible possible counter-logics" (Glynos, 2010 p.31). This logic has a structure: "The logic of fantasy names a narrative structure involving some reference to an idealised scenario promising an imaginary fullness or wholeness (the beatific side of fantasy) and, by implication, a disaster scenario (the horrific side of fantasy). This narrative structure will have a range of features which will vary from context to context, of course, but one crucial element is the obstacle preventing the realization of one's fantasmatic desire" (Glynos, 2010 p.29).

Such narratives are the subject of several research studies of fantasy in the workplace (Costas and Taheri, 2012; Hoedemakers 2007, 2009), which generate their accounts by describing the following: (1) the presentation of an ideal situation (e.g safe healthcare); (2) the obstacle to the realization of that ideal (e.g. human error); and (3) a transgression of that ideal (e.g. unsafe healthcare). These three elements make sense of a situation, imposing a logic on it through a series of equivalences and differences. In our instance of simulation-based medical education: unsafe healthcare is bad healthcare; good healthcare is safe healthcare; the obstacle to good healthcare is human error.

In the critical business literature, fantasy is analysed to generate a critique of dominant norms which are 'suspect' insofar as they deny uncertainty and ambiguity and thereby lock subjects into repetitive cycles of failure and painful affects, notably guilt (Costas and 
Taheri, 2012). To develop our example: the equivalence of good healthcare with safe healthcare makes invisible practitioners' work of judging between risks. Making denied aspects of work visible again is intended to enable employees gain some distance from fantasy narratives, and 'read for difference' (Glynos, 2010); in other words, generate appearances which account for work differently, often by showing the inter-dependence of opposites (e.g. the ideal of safe healthcare as dependent for its perpetuation on its real transgression, notably in the figure of the incompetent doctor). The notion of 'traversing the fantasy' accounts for what happens as a consequence of maintaining a critical distance, identifying a re-organization of the economy of affects and signifers in the workplace, such that, for instance, good healthcare is no longer treated as logically equivalent to safe healthcare, with consequent effects on the distribution of guilt and enjoyment in medical work.

\section{'Simulation studies'}

The second area of literature informing our analysis is less unified, studying simulations from different disciplinary perspectives, including film, internet and technology studies (McGowan, 2013; Dean, 2010; Nusselder, 2013, 2009; Zizek 2004, 1999, 1997; Turkle 1995). Whereas the critical business studies literature focuses on the subjugation of employees, film and internet studies place greater emphasis on consumers' enjoyment and pleasure: "the fantasies accompanying computer technologies boil down to the notion that they offer us means to surpass the limits that reality imposes upon us. The standard fantasy about the new worlds opened up by computer technologies considers them as new spaces where all the old limits might be transcended" (Nusselder, 2009 p.11). Here, 
simulations appear as structured to offer relief from the burdens of reality: what we don't have in reality, we have as a simulation. Consequently, simulations also identify what a burden is, and what is lost or lacking in reality. To develop our example: 'high-fidelity' simulation is described as a way of surpassing the limitations imposed by the burden of reduced training hours (CMO, 2008), a claim which makes the failures and risks presented by medical work appear as temporal phenomena: a lack of time, and thus a loss of experience. Simulation-based medical education then restores what is felt as having been lost.

As in the critical business studies literature, fantasy is treated as a defense mechanism, which protects against the anxiety of uncertainty and ambiguity by staging a scene in which wishes are realized. The question however is (Zizek 1989): whose wishes are they? This is answered in reference to the concept of the Other or the Other's gaze, which describes the perspective from which a scene is staged, such that the riddle of desire (what do I want? what do others want from me?) is answered. The idea of the Other's gaze has been particularly influential in film studies, in exploring the perspective from which characters have to be seen to appear heroic, attractive, horrifying, feminine or masculine, as 'real' men or women, and so on. The Other's gaze is, in this respect, the camera's viewpoint, from which a scene is perceived to make sense, but within which it is not visible.

To take an example close to this paper's subject: in her studies of the Visible Human Project (VHP), Waldby $(2000,1997)$ explores what makes this 'high fidelity' anatomical 
simulation so compelling in medical literature, arguing that its appeal lies in how it shows the body not simply as it is but as it ought to be to a medical gaze: "If the body's life can be described as animated matter, the technology of the VHP can effectively claim to have cancelled out the effects of death by translating the corpse into the simulational space of the virtual screen, with all its powers of complex animation. The VHP partakes of the general repression of death in medical knowledge of life, providing a complex model for the living body which is precisely a reanimated corpse" (1997). The VHP is seen as a pedagogic breakthrough because it shows the body to be manipulable and controllable by medical intervention; as individual and standardised, indistinguishably; and as eternal as software data, and thus no longer the bearer of mortality's burden (by contrast, for instance, to corpses that decay). The ability of the VHP to act as surrogate for the fleshy body is dependent on identifying with this gaze, and the desires it animates.

In this vein of literature, the purpose of analyzing fantasy is to make reality visible as an appearance, rather than an essence; an image or scene which is connected to the experience of pleasure and enjoyment. Simulations are treated not simply as reflections of such affective economies, but also as forms of instruction, teaching how and what to desire. For example, the VHP teaches the features of a desirable body in medicine, notably the qualities of being eternally repairable and not decaying uncontrollably. Analyses of fantasy enable discussion of the terms upon which a representation is seen and taught to be realistic. The notion of 'traversing the fantasy' arises also, and points to the possibility of reconfiguring images to make denied aspects visible, better to respond to them - 'better' here a matter of politics and ethics. 


\section{Studying fantasy in high-fidelity medical simulation}

These two domains of literature provide conceptual resources with which to analyse the use of simulation for professional education. They point to the way in which fantasy can be accounted for in terms of a narrative structure featuring an ideal, its obstacle, and its transgression; as well as a gaze that discriminates between these, in the form of burdens in reality and that which relieves them. The image of the subject is somewhat different in each domain, appearing either as the exploited worker of a capitalist labour market or as an enjoying consumer of limitless capacities. We draw on both domains in part because they offer different kinds of empirical instantiations of the concept of fantasy, with the simulations studies literature exploring medicine specifically, but also because the subject of simulation-based medical education appears to us as neither an exploited worker nor an entertained consumer, but something of both.

Let us now move onto how we arrived at this perception. Between January and October 2012, we observed 30 training courses in the simulation centres of four London teaching hospitals, with a view to describing how teaching and learning happened in practice. The clinicians attending such courses were usually postgraduate, 'trainee' doctors, sometimes also nurses and other health professionals. Each course involved 4-12 trainees, and 4-6 tutors, who were usually senior doctors and nurses. Courses usually lasted one day, and were composed of three elements. First, an introductory lecture describing the purpose of the course. Second, a sequence of scenarios (between 2 and 6), lasting approximately 15 
minutes, and in which 1-2 trainees 'played out' a situation specified by tutors, in a simulated ward which had one-way mirrors and video cameras, so that the other course participants could watch the action. Third, and following each scenario, a 'de-briefing', lasting between 20 and 45 minutes, and in which trainees and tutors discussed what happened. We also interviewed eight trainees, six months after their participation in a course, about the pleasures and frustrations of their work, and whether the course had reorganised these. For this article, we are therefore drawing on field notes, interview transcripts, and the video recordings generated during a course. Ethical approval for the research was granted by Imperial College, London, and funding was provided by the London Deanery.

We have organised our analysis into three parts, identifying patterns across the three elements described above: introductory lectures, scenarios and de-briefing discussions. We will account for each in turn, in the light of the concepts and approaches we have described from the literature on fantasy.

\section{Introductory lectures: simulation as therapy for medical error}

All but one of the courses we observed presented high-fidelity simulation as an occasion on which to learn about a model of medical error called human factors, imported from other 'high-risk industries', notably aviation. This model was said to explain error in two main ways. First, error was a function of faulty systems rather than individuals; second, error was inevitable because humans were fallible. This explanation meant that error 
could be learned from, rather than punished; and could be mitigated by gaining

knowledge of 'non-technical skills'. The following excerpts illustrate how this model was presented:

Human error is responsible for 70 to $80 \%$ [of errors]. So it's not machines going wrong, the drugs are wrong, it's all human error. So 70 to $80 \%$. It's something we need to keep in the back of our minds. We are humans, and we do make mistakes. But it's how we can recognise the risks in the situation that we are in. So we mentioned non-technical skills at the beginning, that we need to deal with a crisis, and the sorts of things that we are going to be looking at in each of the scenarios, although they are all different, is how you cooperate, how you lead, are you aware of the situation that you are in and managing that situation, and how you make decisions. So it's mainly non-technical skills. You'll all be very good at managing a patient who is sick, it's not what we are looking at, although it is part of it. We want to get you thinking about non-technical skills.

(Video transcript, centre 1)

According to this logic, clinical work divides into two domains: the technical/clinical and the non-technical/the interactional. Error is introduced into the clinical domain by means of its instantiation as interactional activity. The catholic resonance of this model of failure is brought out by the title of the medical report that was referenced in introductory lectures: To Err is Human (Institute of Medicine, 1999). Non-technical skills are that which turn inherent human fallibility into a virtue, overcoming the deadlock consequent upon figuring clinical knowledge as failing because of those who enact it. If we look at this model of error in terms of its narrative structure, the ideal is that of a healthy and health-giving system in which the distribution of skills to healthcare workers manages 
risk. The obstacle to this ideal is human fallibility itself: an intractable element identified on the basis of an ethos of resistance to something internal, irredeemable. It is this human factor which leads healthcare astray, causing it to kill rather than cure.

Within this account, simulation is safe because error can be detached from its consequences:

So I often say, when we're running full immersion simulation, particularly with the more junior doctors is that 'make as many mistakes as you want because everyone can learn from it and you can't kill the mannequin'. Yeah? You can make it arrest but you can't kill it whereas if it's a real patient then you can.

(video transcript, centre 2).

The invitation to 'make mistakes' presents high-fidelity simulation as a transgression: because death is expunged, the prohibition on killing patients is removed. Trainees should make mistakes precisely to learn from them, an injunction which presents simulation as a place in which taboos are to be broken. This argument aligns with numerous accounts of 'virtual reality' as a site of perversion in which norms are transgressed (Turkle, 1995), but with the effect of their fiercer enforcement in the 'real world', by virtue of the categorical difference inscribed between the virtual and the real (McGowan, 2013). 
The ethics of this model of error and of simulation are brought into relief by comparing them to earlier accounts of failure in medicine, and by examining the viewpoint from which simulation is consequently perceived to be safe.

In their classic studies of medical error, Bosk (1979/2003) and Paget (1983/2004) depict error as intrinsic to medicine. Bosk's study describes the classifications used by surgeons to identify different types of error, with some treated as constitutive of learning and doing surgery. Paget's treatise shows error to be endemic to medicine, inherent to the experimental effort involved in diagnosis and therapy. In each case, the obstacle to healthcare's efforts to heal are constitutive of its object of knowledge: "medical work does not shape events, but risks a shape for events that are already going wrong" (Paget, 2004 p.128). Accounting for error in terms of human factors shifts the obstacle to the subject who practices medicine; error/failure moves from the deed to the doer. Lack also shifts from medicine as an unpredictable, uncertain practice, to the medical worker who lacks non-technical skills. Simulation then appears safe because such skills can be acquired with certainty - as guaranteed by the safety record of the aviation industry which uses simulation extensively.

Bosk's and Paget's accounts emerge from an identification with professions of medicine: they endeavour to see error as it appears to clinicians in their work, and specifically, in their efforts to address risk in patients' bodies. A scene in which error is staged as tragic exception caused by human fallibility implies identification with another gaze, one which treats medical knowledge as perfectible: error-free when applied correctly. By extension, 
such knowledge manufactures, and also therefore, manages the risks implied by its practice. Paget argues that patients are prone to adopting such a gaze, to deny the burden of anxiety and uncertainty imposed by illness. Dejours' (2008) account of human factors presents it rather as a managerial gaze, in which failure is a problem overcome through employee training. Both of these arguments suggest that human factors teaching can be construed as an invitation to identify with a gaze external to professions of medicine: one which sees such professions as powerful and privileged by dint of the perfection of their scientific knowledge and burdened only by generic fallibility.

Introductory lectures however positioned tutors and trainees differently with respect to how failure could be perceived. Tutors emphasised the democratic consequences of a human factors model, which universalised the propensity to error and suspended the logic by which error equated with inexperience. Trainees however did not take up the invitation to 'make mistakes' enthusiastically: they demonstrated palpable anxiety at the start of courses and often throughout. This anxiety was explained in coffee breaks in terms of failing in the eyes of peers and seniors. This is suggestive of the different ways in which simulation appeared realistic and safe for tutors and trainees. Courses were set up for the benefit of trainees, which excluded the possibility of error being seen in the actions of tutors. Trainees, by contrast, were due to be watched by fellow professionals in order to make their failures perceptible. Error thus appeared on the basis of a lack of credentialised knowledge and experience. The model of 'democratic' fallibility was thus realised in a course structure in which errors could only be seen in the actions of junior individuals - a pattern which repeats the organisation of medical apprenticeship. 


\section{Scenarios: plotting narratives about what clinical work consists of}

Scenarios were referred to in terms of the clinical condition they manifested, such as cardiac arrest, anaphylaxis, or ectopic pregnancy. Dramatised conditions were usually life-threatening. A scenario began with tutors telling trainees a brief narrative giving the patient/mannequin a back-story, such as: 'Mrs Smith has been admitted to A\&E with stomach pain, and you are the first doctor to examine her'. The condition to be simulated was not revealed. The trainees acting in the scenario then entered the simulation room, and examined the mannequin, as well as other information set up by tutors, such as medical notes. A patient's condition invariably deteriorated rapidly, so that the trainee had to call in others to help. All of the scenarios we observed concluded with a resolution of the emergency: the patient's life saved, the condition stabilised.

This narrative organisation meant that presenting problems always had clinical solutions: the obstacle to be overcome was of a clinical nature, calling on the display of specialist clinical knowledge, and leading to clinical outcomes. Such outcomes, such as a stabilised blood pressure, appeared as the consequence of trainees' actions, such as giving fluids. Trainees thereby appeared as heroes in a story that told of the power of clinical intervention: an initial state of relative equilibrium is disrupted by an event (e.g. collapsing blood pressure), the trainee takes action and, by this action, brings the chaos to an end (Todorov, 1977). The hero was played by whichever profession was being trained: courses for anaesthetists idealised anaesthetic intervention; courses for surgeons idealised 
surgical intervention; courses aimed at junior medical trainees demonstrated the power of their professional capacity to save patients, and featured seniors and nurses as secondary characters within the drama. One course only departed from this trope: aimed at nurses, participants were called on to respond to multiple, relatively stable patients (rather than a single critically ill one) and ongoing obstacles that were not resolved. By virtue of this narrative structure, the role of the herculean hero was replaced by that of the sisyphean worker, a difference which perhaps throws some light on the different organisation of desire in medicine and nursing.

Although scenarios were differentiated by the clinical classification of the obstacle, the element common to all of them was the threat of death. Always present in the wings, death however never entered the stage, a narrative convention which tutors described as 'the manikin never dies'. The removal of death's burden echoes Waldby's (1997) claim that in marking medicine's point of failure, death is repressed from medical simulation. However, whereas in Waldy's anatomical simulators death was not represented, it appeared in our study as foreclosed transgression: always there, and always pre-empted. The nightmare of killing patients was repeatedly envisaged and repeatedly dissipated.

Ahmed's (2009) articulation of happiness and its role in 'suturing' a collective is perhaps helpful here: she refers to happiness as an ideal which establishes consensus on what a 'we' should strive for, illustrating this through the image of the happy family. By representing an object cause of happiness, the image of the happy family enforces heterosexual norms, by making non-heterosexual bonds appear necessarily unhappy. In 
representing patient survival as the collective object of clinical desire, and such survival as a function of 'teamwork', the solidarity of work appeared structurally, narratively, in opposition to the death of patients: patients survived because clinical teams happily bonded.

In presenting patient survival, professional belonging and clinical capacity as equivalent, the narrative conventions of scenarios reflected trainees' accounts, generated during interviews, of what made clinical work enjoyable:

You may sort of feel embarrassed to say it, but I do quite like the adrenalin when you've got somebody, and it's three in the morning, and they're about to die and you're trying to stop them about to die, I quite like that.

(Angela, medical trainee)

However, the desirability and excitement of near patient death contrasts with how trainees portrayed their usual work routines:

I remember the initial disappointment of becoming a doctor, and it's your first year as a doctor, and you think you're going to be seeing patients and actually a lot of what you do is being delegated these menial administrative tasks.

(Tracy, medical trainee)

The imagery of clinical power overcoming obstacles also contrasts with how trainees represented the frustrations of their work: 
All specialities come with some conditions which slightly fill you with a sense of futility, that you're not really doing anything. So $[\ldots]$ people with alcoholic liver disease. You work really hard and you do all the exciting stuff to get them better but they'll keep drinking and they'll come back again.

(Peter, medical trainee)

These accounts of the pleasures and frustrations of clinical work are suggestive of the enjoyment offered by simulation scenarios: they dramatised narratives of clinical (and trainee) capacity to shape events, making imperceptible menial administration and futile expert action. These consequently appeared as external to 'real' clinical work.

We discussed above the argument in the 'simulation studies' literature that the pleasure they afford and the realism they are attributed stems from the way they remove burdens which limit the scope for self-realising action in 'reality' (Nusselder, 2009; Zizek, 2013). The narrative structure of scenarios, including the choice of obstacle confronting the hero (which for instance does not require him/her to address intractable welfare needs or operate marginally in bureaucratic organisations) shows both how they appeared realistic (showing real clinical action, in a way parallel to how films generate pleasure by showing real men or real romance); as well as how they functioned pedagogically, teaching not merely skills, but their meaning within a vision of what clinical work should, ideally, consist of.

\section{De-briefing: making error and failure explicable}


Scenarios were followed by group discussions arranged in a large circle. Tutors started by asking the group to describe what happened, often requesting that those who had participated in a scenario withhold their input initially. This was intended to collectivise the experience, and also acknowledged that such participants were often lost for words. In describing 'what happened', trainees and tutors constructed a narrative organised by causes and reasons, decisions and strategies, and historicised through the use of the past tense, which made events perceptible through a lens of hindsight, in relation to what they eventually led to.

Accounting for what happened however positioned trainees and tutors differently. Trainees who had participated in a scenario often referred to what they should have done, what they had not seen or known, what they had not done well: the scenario, in its retrospective signification, was narrativised in the tragic genre of a tale of regret and missed opportunities. By contrast, tutors' interventions - notably, the frequently asked 'so, what could you have done differently?' - portrayed the scenario as one possible performance among multiple others, imbued with what Murray (1997) describes as the 'malleability' of the comedy genre, in which mistakes are retrievable and stories can be started over and reach a different resolution - foreclosing, for instance, the finality of death.

The difference in genre points to a difference in gaze. In manifesting guilt in the wake of failure, trainees identified with a gaze that knows and passes judgement on correct and incorrect action, innocent and culpable people. This is suggestive of a desire to know, 
with certainty and without ambiguity, one's capacities as a clinician; knowing, for sure, whether one's actions are correct or erroneous. In describing non-technical skills as indeterminate, tutors identified with (and explicitly named) a 'non-judgemental' gaze from whose comic perspective failure was an occasion for bonding. One might argue then that trainees' confessions did not address an actual gaze - that of tutors - but an imagined one, with much greater authority. This account was offered to us by several tutors who characterised trainee interventions in de-briefings as repeated requests for reassurance.

Such an account neglects two phenomena. The first is the explanation for failure given by tutors to trainees, which established a de facto guilt by virtue of the human factor. The second is the repeated offer of reassurance given by tutors to trainees on the quality of their performance. Although simulation courses were described as occasions for 'nonjudgemental' teaching interventions, the usual pattern of exchanges consisted of: tutor initiation of the discussion; trainee response; tutor's positive judgement. As an example:

$\begin{array}{ll}\text { Tutor } 1 & \text { So describe what happened? [...] } \\ \text { Trainee } & \text { I think the things I know, I notice this in myself in my } \\ & \text { clinical practice is my...I come up with a clear plan } \\ & \text { and can't communicate it without/I sort of chop and } \\ & \text { change a lot } \\ \text { Tutor } 1 & \text { You were very clear. First you wanted [this } \\ \text { Trainee } & \text { Yes], and then I wanted that } \\ \text { Tutor } 1 & \text { And then you changed your tack [...] } \\ \text { Trainee } & \text { And then quite early on I had to have a quite grumpy } \\ & \text { conversation with someone on the phone. I was }\end{array}$


probably a bit rude, and I do apologise for that [...]

Tutor 2 And you were very succinct in stating he's sick, we haven't done anything, we are resuscitating, and he's not going to go anywhere, so I think that was really good

Tutor 1 I think you were really clear, I think you were assertive $[\ldots]$

Tutor 2 I thought you did that very well, because you were assertive

Tutor $1 \quad$ You were very assertive, I thought it was very good

Tutor 2 And the whole point of your focus was your patient

That was very apparent. And that you really didn't have time to talk. And you can have all of this administrative conversation in the background later on. That was done very effectively

(video transcript, centre 2)

The extract illustrates a discursive pattern of re-signifying confessed failures: so, 'chopping and changing' is re-signified 'changing tack', 'rude' is re-signified 'assertive'. The pattern illustrates how human factors were taught: namely, by initiating an account of a guilty self, and then re-signifying it to make it show non-technical capability. The substantive content of 'non-technical skills' was thus the negation of guilt. Tutors' comedic interventions might also be understood in terms of such a negation. Negation however implies recognition of a positive form (Kress and Hodge, 1979): trainees' anxiety was consequent, we would argue, upon this recognition. 


\section{Fantasising collective action to rescue patients}

To explore the ethical implications of this negation, we would like to compare the discursive pattern above to an exception within our fieldwork. It occurred during a course organised to remedy the high number of 'failure to rescue" ${ }^{1}$ incident reports generated by one ward. All ward staff had been invited to attend, although on the day of our visit, participants consisted exclusively of nurses. During the introductory lecture, a tutor (a doctor) said that the course was not about punishing poor performance but learning to respond better to an emergency. Several participants responded sceptically:

\footnotetext{
So what are you going to do if you come round to my ward and I have seven patients to look after, two post-ops, and no $\mathrm{HCA}^{2}$. What are you going to say or do?
}

(Field notes, centre 4)

The tutor responded that 'hospital management', in the form of the deputy director of nursing, had also been invited, so that she could learn about the problems staff were experiencing, and support them in overcoming these. Several participants responded that 'their' problems were caused by management's cost-cutting exercises.

The first of several scenarios then took place. These were however perceived differently than on other courses: they simulated something different. The following extract from a group discussion illustrates this:

Tutor 1 The problem is, Sally, you didn't tell your team-mates

you had the sickest patient. I was just interested in 
knowing - it's not a criticism - why you felt you had to cope on your own?

Nurse 1 That's what I do on the ward

Tutor $1 \quad$ You might be stuck in a ward when you are trying to

cope and you don't go and get help?

Nurse 1 I'm just re-living yesterday, it's constantly juggling. I

find it heavy-going, but I don't want to seem I'm not

coping with it

Nurse 2 We need to re-design the system so that nurse leaders

don't get attached to particular beds and oversee the

whole ward [there follows an extended discussion

about whether this system is in fact already in place but

undermined by staff shortages]

Tutor $1 \quad$ You need to think as a group how you want to deal with these situations. You need to have agreement on this, as a unit

(Field notes, centre 4)

Sally's failure/error here appears as common practice on the ward. Nurse 2's intervention, for instance, treats Sally's failure to call for help as indicative of the division of labour on the ward, a move corroborated by Tutor 1's final contribution. What the scenario shows, therefore, is how work is done, including the feelings associated with this ('juggling, heavy going'). What it does not appear to show, as on other courses, is Sally's (inadequate) clinical capability or inherent human fallibility.

In this and other group discussions on the day, scenarios were treated by participants as ways of showing management, as well as each other (note above the 'I don't want to 
seem I'm not coping with it'), the realities of work, in contrast to its prescribed representation; for instance, subsequent to the extract quoted above, there was extended discussion about the impossibility of completing the 27-point admissions checklist within allocated time. This made Sally's failure illustrative of an antagonism between resources and prescribed work tasks, and signified the checklist as the expression, but not the realisation, of the ideal to make patients safe. The obstacle to such an ideal shifted from human fallibility to the multiple and conflicting desires within healthcare. The transgression of that ideal appeared as unrealised protocols consequent upon conflicting priorities, rather than employee ignorance or incapability.

This transformation in what could be seen in a scenario - the basis on which it appeared realistic - illustrates aspects of the Lacanian notion of 'traversing the fantasy'. The introductory lecture and subsequent group discussions staged a conflict between two narratives for failed rescue: 'management only care about cutting costs' versus 'nurses lack non-technical skills'. The re-enactment of clinical work made visible the gap between each explanation for failure and inarticulated work/management practices: it made visible what each explanation endeavoured to hide, such that the hospital's budget had been cut and that staff numbers had been reduced. Real antagonisms within healthcare provision became speakable, making it necessary for nurses and tutors to loosen attachments to fantasised narratives about failure.

The notion of traversing the fantasy implies constructing alternative/better fantasies, rather than abandoning fantasy altogether (Zizek, 2013). So what were the two fantasised 
narratives for failure replaced with? Amid discussions about whose performance was to blame, tutors' interventions evoked the possibility of finding better ways of managing antagonisms between work tasks. In the extract above, for instance, the tutors do not offer absolution, but rather ask the group to find, collectively, responses to the realities that the scenario showed. This request was resisted several times with counter-claims that management had this responsibility, but statements were also made describing intentions to review the division of labour with respect to specific tasks; the ability and right to do this was thereby also claimed (Rancière, 2004). The fraughtness of such discussions demonstrates the work (in the psychoanalytic as well as the everyday sense) involved in constructing the fantasy of collective, cooperative, team working (Dejours, 2009). Although agreement was not reached on the day of our visit, there was promise in fantasising the division of labour as a collective achievement, not only because it offered a practical example of 'more democratic healthcare', but also because it grappled with the problem of failed rescue more substantively than fantasies of human fallibility and negated guilt.

\section{Conclusion: re-thinking the relationship between simulation, fidelity and learning}

In this paper, we have endeavoured to show that high-fidelity simulation does not simply denote clinical practice but mythologises it (Barthes, 1957, 1961): it is not so much analogous to reality, mechanically reproducing it, but rather constitutes a commentary on clinical practice, teaching values, reasons and desires about how such practice ought to be seen and felt. It is insofar as participants identify with such a perception that simulation 
appears faithful to reality. This argument has implications for considering the ethics of simulation, raising questions about how it is implicated in teaching professional ideals and identifying the obstacles to their realisation, as well as the burdens of clinical work.

Treating simulation as a mythology might be read as a criticism of its educational value: a dismissal of its claim to realism and an identification of its deceptive illusions. Such a reading treats simulation as a lie, in the Platonic sense of a simulacrum, and/or as an occasion for ideological revelation, showing how far the forgery has strayed from its authentic original. Both moves assume that reality can be seen and known directly, unmediated by fantasy. We have drawn on the Lacanian notion of fantasy to avoid this reading of our argument, and to treat fantasy as that which enables reality to make sense - something one can be faithful to - rather than which leads away from it.

The concept of fantasy does however denaturalise reality, pointing to how it emerges affectively, by excluding, abjecting, and papering over contradictions. In the first part of the analysis, we focused on how an account of human factors assigned risk to human fallibility and systems design, excluding it thereby from clinical work and patients' bodies. Compared to earlier sociological accounts of medical failure, the human factors narrative shifted error's location from the practice of clinical work to the practice of clinical workers. This was justified in terms of patient safety, making error into a redeeming occasion for learning rather than the endlessly and unproductively repeating nightmare of killing patients. 
We have argued that this way of making error into a phenomenon one can learn from made practitioners guilty by default. By implication, also, it made them not responsible. It is in the light of this account of a perfectible science degraded by the fallibility of its human yet heroic practitioners that we interpret trainees' anxiety during simulation courses, as well as the guilt articulated in de-briefings, which was invariably accompanied by a denial of responsibility: indeed, whenever errors were evoked (almost exclusively by trainees on their own performance), there followed shortly after a discussion of their causes in the limitations of the technology. This raises a question about what was learned from error on simulation courses.

What was stated to be learned was how to avoid or mitigate errors in 'real' clinical practice. This treats error as a matter of consensus: it is there for all to see and infer the lesson from. Our account of the 'failure to rescue' course shows rather the identification of error, and the account of clinical reality, to be contested social practices - a claim often made in literature on error in 'real' clinical practice (Waring, 2009). The work and frustration we witnessed in explaining 'failure to rescue' points to what was avoided on most courses, in which emphasis was placed by tutors on identifying and praising 'nontechnical skills' and emphasising realism, rather than discussing failure. The ethics of simulation have been justified in terms of pre-empting the social antagonism associated with medical error and its consequences, and which make error difficult to learn from (Ziv et al, 2003); we would however argue that such antagonism was pre-empted not so much by the cancellation of error's effects as by the marginalisation of considerations such as whose view of reality and error was represented in a simulation, whose was 
excluded, and according to which principles and pleasures had clinical practice been selectively 'sieved' for its simulation.

We started this paper by describing our aim in terms of closing the gap between the original and its forgery and encouraging 'more democratic healthcare'. These aims are closely related, in treating reality as contingent appearance rather than given essence: a phenomenon subject to disagreement rather than expert consensus. Defending these aims does not involve giving up on reality and sinking into relativism and 'mere' fantasy, but rather putting questions about what constitutes reality at the centre of simulation's pedagogic practice, such that disagreements about what makes clinical practice good, better or fail, meaningful or meaningless, are visible and articulable. Our account of the 'failure to rescue' course is suggestive of how this might be achieved, as well as the work involved in bringing fantasies to light through the identification of difference within reality; within the multiple and conflicting narratives about what is desirable in healthcare. It shows what scope there is for simulation to change from serving as an ante room prior to entry into a given reality, to an occasion on which to dramatize and 'work through' the fantasies which make clinical practice meaningful, as well as the obstacles which threaten this. In this respect, we would argue for an ethics of simulation based on its instantiation of a distinct regime of visibility, in which the meaning and organisation of work is subject to collective deliberation, rather than on its promise of protecting patients from doctors. This regime can be imagined as allowing fantasies to be adjusted and re-framed, to address repeated and painful failure, including the death of patients. 


\section{Endnotes}

1. failure to rescue' is a category within the hospital's taxonomy of errors which refers to an event in which healthcare workers failed to respond appropriately to a rapidly deteriorating patient, who then died.

2. HCA stands for healthcare assistant

\section{References}

Ahmed, S. (2009) Affect aliens: Happiness as a Cultural Politics. In: J. Satterthwaite, H. Piper and P. Sikes (eds.) Power in the Academy. Stoke-on-Trent: Trentham books, pp. 117-134.

Barthes, R. (1957) Mythologies. Paris: Editions du Seuil.

Barthes, R. (1961) The photographic message. In: Barthes, R. (ed.) Image, Music, Text. London: Fontana, 15-31.

Bosk, C. (1979/2003) Forgive and remember: managing medical failure. $2^{\text {nd }}$ edition. Chicago: Chicago UP.

Cederstrom, C. and Hoedemakers, C. (Eds.) (2010) Lacan and Organization. London: May Fly Books.

CMO (Chief Medical Officer) annual report (2008) Safer medical practice: Machines, manikins and polo mints. London: Department of Health.

Costas, J. and Taheri, A. (2012) The return of the primal father in postmodernity? A Lacanian analysis of authentic leadership. Organisation studies 33(9): 1195-1216. 
Curran I. (2010) Innovation in Education - the engine for change. Conference presentation at the 3rd Annual STeLI Conference, London. March, Royal College of Physicians.

Dean, J. (2010) The Real Internet. International Journal of Zizek Studies 4(1). http://zizekstudies.org/index.php/ijzs/article/view/242/322

Dejours, C. (2008) Le Facteur Humain. Paris: PUF.

Dejours, C. (2009) Travail et sexualite 1. Paris: PUF.

Gaba, D. M. (2004) The future vision of simulation in health care. Quality and Safety in Health Care 13: i2-i10.

Glynos, J. (2008) Ideological fantasy at work. Working paper in ideology in discourse analysis, 23. http://www.essex.ac.uk/idaworld/working_papers.html

Glynos, J. (2010) Lacan at work. In: Cederstrom, C. \& Hoedemakers, C. (eds.) Lacan and Organization. London: May Fly Books, pp. 13-58.

Glynos, J. (2011) On the ideological and political significance of fantasy in the organization of work. Psychoanalysis, culture and society 16(4): 373-393.

Hoedemaekers, C. (2007) Performance, Pinned Down: A Lacanian Analysis of Subjectivity at Work. Rotterdam: ERIM.

Hoedemaekers, C. (2009) Traversing the empty promise: management, subjectivity and the Other's desire. Journal of Organizational Change 22(2): 181-201.

Institute of Medicine (1999) To Err is Human: Building a Safer Health System. Atlanta: National Academies Press. 
Issenberg, S. B., McGaghie, W. C., Petrusa, E. R., Gordon, D. L., and Scalese, R. J. (2005) Features and uses of high-fidelity medical simulations that lead to effective learning: a BEME systematic review. Medical Teacher 27(1): 10-28.

Johnson, E. (2008) Simulating medical patients and practices: bodies and the construction of valid medical simulators. Body and Society 14(3): 105-128.

Johnson, E., and Berner, B. (eds.) (2012) Technology and Medical Practice: blood, guts and machines. Farnham: Ashgate.

Kress, G. and Hodge, R. (1979) Language as Ideology. London: Routledge \& Kegan Paul Ltd.

Lacan, J. (2007) The ethics of psychoanalysis. London: Routledge.

Lapping, C. (2013) Institutional accountability and intellectual authority: unconscious fantasies and fragile identifications in contemporary academic practice. In: C. Maxwell and P. Aggleton (Eds.) Privilege, agency and affect: understanding the production and effects of action. Basingstoke: Palgrave Macmillan, pp. 88-105.

McGowan, T. (2013) Virtual freedom: the obfuscation and elucidation of the subject in cyberspace. Psychoanalysis, culture and society, 18(1): 63-70.

McNaughton, N. (2012) A Theoretical Analysis of the Field of Human Simulation and the Role of Emotion and Affect in the Work of Standardized Patients. Ontario Institute for Studies in Education, University of Toronto.

Murray, J. (1997) Hamlet on the Holodeck: The Future of Narrative in Cyberspace. Cambridge: MIT Press.

Nusselder, A. (2009) Interface fantasy: a Lacanian cyborg ontology. Cambridge: MIT press. 
Nusselder, A. (2013) Twitter and the personalisation of politics. Psychoanalysis, culture and society 18(1): 91-100.

Paget, M. A. (1983/2004) The unity of mistakes. Philadelphia: Temple UP.

Pelletier, C. (2009) Education, Equality and Emancipation: Rancière's critique of Bourdieu and the Question of Performativity. Discourse: studies in the cultural politics of education 30(2): 137-150.

Pelletier, C. (2012) No time or place for universal teaching: The Ignorant Schoolmaster and contemporary work on pedagogy. In: J. P. Deranty and A. Ross (Eds) Jacques Rancière and the contemporary scene. London: Continuum, pp. 99-116.

Rancière, J. (1987) Le maitre ignorant. Paris: Fayard.

Rancière, J. (2004) The politics of aesthetics. London: Continuum.

Rancière, J. (2004a) The Philosopher and His Poor. Durham: Duke University Press.

Stavrakakis, Y. (2008) Subjectivity and the Organized Other: Between Symbolic Authority and Fantasmatic Enjoyment. Organization Studies 29(7): 1037-1059.

Stavrakakis, Y. (2010) Symbolic Authority, Fantasmatic Enjoyment and the Spirits of Capitalism: Genealogies of Mutual Engagement. In: Cederström, C. and Hoedemaekers, C. (eds.) Lacan and Organisation. London: MayFlyBooks, pp. 59-100.

Taylor, J. S. (2011) The moral aesthetics of simulated suffering in standardised patient performances. Culture, medicine and psychiatry 35(2): 134-162.

Todorov, T. (1977) The poetics of prose. Ithaca: Cornell UP. 
Turkle, S. (1995) Life on the screen: identity in the age of the internet. New York: Simon and Shuster.

Waldby, C. (1997) Revenants: the Visible Human Project and the Digital Uncanny. Body and Society 3(1): 1-16. http://wwwmcc.murdoch.edu.au/ReadingRoom/VID/Uncanny.html

Waldby, C. (2000) The Visible Human Project: Informatic Bodies and Posthuman Medicine. London: Routledge.

Waring, J. J. (2009) Constructing and re-constructing narratives of patient safety. Social Science and Medicine, 69(12): 1722-1731.

Ziv, A., Wolpe, P. R., Small, S. D. and Glick, S. (2003) Simulation-based medical education: an ethical imperative. Academic medicine 78(8): 783-8.

Zizek, S. (1989) The sublime object of ideology. London: Verso.

Zizek, S. (1997) The plague of fantasies. London: Verso.

Zizek, S. (1999) Is it possible to traverse the fantasy in cyberspace? In: E. Wright and E. Wright (eds.) The Zizek reader. Oxford: Blackwell Publishing, pp. 102-124.

Zizek, S. (2004) The Cyberspace Real. www.egd.edu/faculty/zizek/zizek-the-cyberspacereal.html.

Zizek, S. (2013) Less than nothing: Hegel and the shadow of dialectical materialism. London: Verso. 